Copyright 2021 Society of Photo-Optical Instrumentation Engineers (SPIE). One print or electronic copy may be made for personal use only. Systematic reproduction and distribution, duplication of any material in this publication for a fee or for commercial purposes, and modification of the contents of the publication are prohibited.

This is the accepted version of the paper available at https://doi.org/10.1117/12.2594266 


\title{
Recommendations on the viewing distance of light field displays
}

\author{
Peter A. Kara ${ }^{\mathrm{a}}$, Attila Barsi ${ }^{\mathrm{b}}$, Roopak R. Tambolic, Mary Guindy ${ }^{\mathrm{b}}$, Maria G. Martini ${ }^{\mathrm{d}}$, \\ Tibor Balogh ${ }^{\mathrm{b}}$, and Aniko Simon ${ }^{\mathrm{e}}$

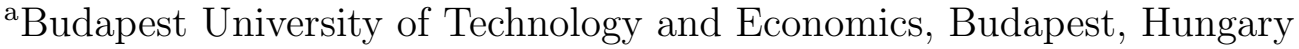 \\ ${ }^{\mathrm{b}}$ Holografika, Budapest, Hungary \\ 'Indian Institute of Technology Hyderabad, Kandi, India \\ ${ }^{\mathrm{d}}$ Kingston University, London, United Kingdom \\ eSigma Technology, Budapest, Hungary
}

\begin{abstract}
Light field visualization is an emerging 3D technology that does not rely on viewing devices, such as special glasses. Without the need for such additional devices, multiple users may simultaneously enjoy the glasses-free 3D experience from a virtually infinite number of viewing positions, as the motion parallax is continuous and smooth. Although this immensely valuable property of light field displays does enable a great deal of viewing freedom, the proper perception of the visualized content still depends on the position of the observer. Position in this context determines the distance and the angle of the observer, with respect to the screen of the display. The validity of the latter is relatively straightforward to assess: if the observer is viewing the screen from inside the angle set by the field of view of the display, then the content shall be seen from its perceptually correct angle. However, the fundamental issue with the underinvestigated topic of viewing distance is that light field visualization may lose its 3D nature if viewed from a far-away position. This is due to the fact that in such a case, a single point of the screen may emit identical rays of light towards the two pupils of the observer. Therefore, the deficiency of disparity, the lack of distinct rays may lead to a $2 \mathrm{D}$ visual experience, nullifying the core scientific contribution of this technology. Of course, moving sideways in front of a horizontal-only-parallax display may, in fact, induce a 3D experience via the natural sense of parallax, but it should be applicable regardless of movement. Needless to say, in a multitude of professional environments, the users of such displays are not expected to be highly mobile during the work-related utilization of the technology. However, at the time of this paper, merely a few publications of the scientific literature address the topic of viewing distance, and standardization efforts are still in a rather early phase. Summa summarum, light field displays need clear guidelines with regard to viewing distance, which may benefit both the manufacturers and the users. Such guiding principles may make development and production more cost-efficient and user-oriented, and may contribute to the increased efficiency of usage-focused display design. In this paper, we provide a series of recommendations on the viewing distance of light field displays. The displays are separately analyzed within the context of their own use cases, taking into account the key performance indicators of both the apparatus and the visualized content, the various environmental conditions, as well as the relevant use-case-scenario-specific necessities and the professional requirements. The investigated use cases include medical imaging, telepresence, resource exploration, prototype review, training and education, gaming, digital signage, cinematography, cultural heritage exhibition, air traffic control and driver assistance systems.
\end{abstract}

Keywords: Light field, 3D perception, viewing conditions, key performance indicators, user-centric design

Further author information: (Send correspondence to Peter A. Kara)

Peter A. Kara: E-mail: kara@hit.bme.hu

Attila Barsi: E-mail: a.barsi@holografika.com

Roopak R. Tamboli: E-mail: ee13p0008@iith.ac.in

Mary Guindy: E-mail: m.guindy@holografika.com

Maria G. Martini: E-mail: m.martini@kingston.ac.uk

Tibor Balogh: E-mail: t.balogh@holografika.com

Aniko Simon: E-mail: aniko.simon@sigmatechnology.se 


\section{INTRODUCTION}

Light field is often perceived as the long-term future of 3D display technology. Its potentials fundamentally originate from the fact that no viewing devices (e.g., glasses or helmets) are required to enjoy the use cases of 3D visualization. While the same can be stated about conventional multiview displays, it is a crucial added value that light field displays provide smooth and continuous motion parallax within the field of view (FOV).

The FOV determines the angle of the valid viewing area (VVA). Within the VVA, any reasonable number of viewers (i.e., as many people can fit into the area) may simultaneously observe the visualized content. Yet the FOV only determines the angle of the VVA, and not its maximum viewing distance with respect to the screen of the display.

This distance is solely influenced by the angular resolution of the display - another important key performance indicator (KPI) of light field visualization. ${ }^{1}$ In case of $2 \mathrm{D}$ displays, the recommended viewing distance is commonly based on the height of the screen, denoted by $H$. In the scope of this paper, $H$ is not considered, since the 2D-equivalent spatial resolution is not as vital to the overall perceived visualization quality of light field displays as angular resolution is. ${ }^{2}$

The rule of thumb within the scientific community is that in order to achieve 3D sensation during the observation of light field content, the two eyes of the viewer must be addressed by two distinct light rays, coming from the same point on the screen of the display. In case of horizontal-only parallax (HOP) displays, the better the angular resolution is (i.e., the lower the number expressed in degrees is), the farther viewing distances are supported and thus the greater the VVA is.

However, the rule of thumb initially emerged for a static model (i.e., in which the observer is perfectly still), and movement may affect the 3D sensation and the perception of angular quality. ${ }^{3}$ For example, sideways movement in front of a HOP display may induce 3D sensation via the correctly changing content angle - that is realistically matching the viewing angle - and it may also compensate the angular disturbances of visualization. Furthermore, certain use cases have different criteria regarding visualization quality and 3D perception than others, and thus viewing distance must be taken into consideration when creating the user-centric designs of the light field use cases of the future.

In this paper, we propose a set of recommendations for the viewing distance of light field displays. The investigated use cases are addressed separately, and their associated situational and professional requirements are taken into consideration as well. The recommendations are provided in the form of intervals, which adjust the threshold distance set by the rule of thumb.

The paper aims to cover the most relevant use cases; however, it is not the goal to include every single potential use case in our analysis. For example, real-time military sandbox and devices of home entertainment are not considered. Although the latter is a particularly interesting utilization context of light field technology, it is not likely that such consumer-grade displays shall emerge in the near future and appear on the market at affordable price ranges. Furthermore, the paper focuses on projection-based solutions, such as the back-projection and front-projection HoloVizio displays (e.g., the $722 \mathrm{RC}^{4}$ display and the $\mathrm{C} 80^{5}$ cinema system, respectively).

The remainder of the paper is structured as follows: Section 2 briefly reviews the published scientific works and the standardization efforts that are related to the topic of the paper. Section 3 introduces the different projectionbased light field display types and their relevant considerations. Section 4 provides the list of recommendations, separately for each investigated use case. Section 5 concludes the paper.

\section{RELATED WORK}

\subsection{Scientific literature}

As real light field displays have already been around for over a decade now, there have been numerous subjective tests on such devices, during the planning of which, the matter of viewing distance was evidently considered. In this part of the section, the related scientific literature is analyzed - with particular focus on viewing distance, of course. 
The work of Kara et $a l .{ }^{6}$ directly addresses binocular disparity and presents a study on the theoretical maximum viewing distance for light field displays. It builds on the rule of thumb, which is

$$
D_{V}=\frac{D_{E}}{\tan (A R)}
$$

where $A R$ is the angular resolution of the display, $D_{E}$ is the average interpupillary distance (commonly measured to be $6.5 \mathrm{~cm}$ ), and $D_{V}$ is the viewing distance at which the $3 \mathrm{D}$ experience is still supported.

However, prior to the subjective tests, there have been a number of debates on how exactly the rule of thumb is supposed to be interpreted. Practically, if the viewing distance is smaller than the ratio presented on the right side of Equation 1, then the two eyes (i.e., pupils) of the observer can be addressed by two distinct light rays originating from a given point of the screen. Yet on its own, this does not specify how 3D sensation is affected.

One idea was that viewing a light field display at $D_{V}$ distance would result in a near-perfect $3 \mathrm{D}$ experience, while it was also an interpretation that any observation at this distance and beyond would make light field visualization appear to be flat 2D. However, the results of the work clearly indicate that $D_{V}$ distance is the threshold at which human observers are unable to decide whether the visual experience is more 2D or 3D. Viewing the display from closer positions gradually increases the 3D sensation, and the relationship is close to linear.

The relevant research efforts of the scientific literature are summarized in Table 1. AR denotes the angular resolution of visualization, and provides the value in degrees. If the content $A R$ was worse (i.e., higher numeric value) than the display $A R$, then evidently the content $A R$ is reported, since that is the actual angular resolution of light field visualization. $D_{V}$ real and calc. indicate the actual viewing distance used in the work and the $D_{V}$ value calculated from Equation 1 in centimeters, respectively. The final column, "Movement", reports whether the observers were moving or not during the experiment, and if yes, it specifies the direction(s) of motion. In some of these works, multiple values or value ranges were used (e.g., the content was visualized with different $A R$ values or the observer could change the viewing distance within an area); the minimum and the maximum values of these parameters are indicated in the table. It is important to highlight that viewing distance intervals do not necessarily refer to observer motion.

\begin{tabular}{|c|c|c|c|c|}
\hline Publication & $A R$ [deg] & $D_{V}$ real $[\mathbf{c m}]$ & $D_{V}$ calc. $[\mathbf{c m}]$ & Movement \\
\hline Ahar et al. ${ }^{7}$ & 1 & 280 & 372 & None \\
\hline Cserkaszky et al. ${ }^{8,9}$ & $0.5-2$ & $460-650$ & $186-745$ & Both \\
\hline Cserkaszky et al. ${ }^{10}$ & $0.5-4.5$ & 460 & $83-745$ & Sideways \\
\hline Darukumalli et al. ${ }^{11}$ & 0.5 & 460 & 745 & None \\
\hline Dricot et al. ${ }^{12}$ & 0.5 & 600 & 745 & None \\
\hline Jones et al..$^{13}$ & 1.25 & 100 & 298 & Not defined \\
\hline Kara et al..$^{3,14,15}$ & $0.5-1$ & 460 & $372-745$ & Sideways \\
\hline Kara et al. ${ }^{16}$ & $0.5-2.25$ & $460-560$ & $165-745$ & None \\
\hline Kara et al..$^{17}$ & 1.5 & $30-500$ & 248 & Both \\
\hline Kawakita et al. ${ }^{18}$ & 0.25 & 550 & 1517 & Both \\
\hline Lee et $a l .{ }^{19}$ & 2.6 & 120 & 143 & None \\
\hline Petrov et $a l_{.}{ }^{20}$ & 2.92 & $100-500$ & 128 & Sideways \\
\hline Tamboli et al. $^{21-23}$ & 1 & 244 & 372 & None \\
\hline
\end{tabular}

Table 1. Values of angular resolution and viewing distance in the related scientific literature.

In most of these works, the actual $D_{V}$ is below the calculated one. It should be noted that in certain works, the content $A R$ is reduced but the viewing distance is unchanged, in order to assess the overall subjective visual quality degradation. Furthermore, there are several other works within the scientific literature that could also be included in this brief review, but unfortunately, data on the viewing conditions is either missing viewing distance or it is not presented at all. 


\subsection{Standardization}

At the time of this paper, there is no published international standard or recommendation that addresses the viewing distance of light field display systems. However, the upcoming IEEE P3333.1.4*, the Standard for the Quality Assessment of Light Field Imaging, is aiming to fill this gap. As it is stated in the project details:

"Visual environment characteristics and viewing conditions are also part of the scope of the standard, including viewing distance, viewer position, viewing freedom, and display characteristics."

\section{DISPLAY-SPECIFIC CONSIDERATIONS}

\subsection{Back-projection displays}

The screens of back-projection displays - as the name suggests - receive the incoming light rays from the side opposite to observation. This concept is analogous to conventional television sets, and similarly to regular TVs, one may come as close to the screen as one wishes. Hypothetically, there should be no such thing as "too close" in case of a back-projection light field display. However, let us imagine a large, a very large display (e.g., one that is several meters wide). Let us also assume that this display has a great depth budget - particularly positive depth budget - as well, and that the visualized content fully utilizes this property of the display. Observing this display from a position that is closer to the screen than the extent of the depth budget would result in invalid visualization (i.e., parts of the content would be missing). Therefore, it is a reasonable limitation to have a viewing distance that is not closer to the screen of the display than the positive depth budget.

Additionally, in case of back-projection HOP displays, one must keep in mind that there is no variation in light rays along the vertical axis; practically, they can be considered 2D along that direction. Furthermore, projectors optimize visual sharpness for the plane of the screen. Hence, in order for the observers to maintain a sharp vision of the visualization, a minimal distance from the screen should be kept (at least around $30 \mathrm{~cm}$ in case of a PC-monitor-sized screen). Moreover, known viewing-distance-dependent, content-related rendering-dependent distortions are out of the scope of this paper.

However, these considerations do not apply to super resolution full-parallax (FP) displays. Super resolution refers to an angular ray density during which at least two distinct rays address a single pupil, with respect to a given point on the screen. It enables proper depth vision, meaning that the eyes of the observers may focus on different parts of the content at different depth levels of the visualized light field. Generally, in every other case, the eyes always focus on the plane of the screen. In case of such ray density along the two axes, one may actually observe the content from any given distance without the disturbance of the perceived 3D quality.

\subsection{Front-projection displays}

In addition to what applies to back-projection displays, the utilization of front-projection displays is also constrained by the positioning of the optical engines (i.e., the array of projectors). Since the projectors and the viewer(s) are on the same side of the screen of the display, it is essential that no viewer intersects the light rays coming from the projectors. Should that happen, the intersecting part of the individual would result in visual information loss that is proportionate to the amount of obstructed rays (i.e., the individual would cast a shadow on the screen). Therefore, this scenario must be prevented by setting a proper minimum viewing distance, that even accounts for exceptionally tall observers. It may be a rather strict approach to exclude the area between the projectors and the screen from the VVA. In fact, in case of large systems with high-end properties, the size of this area may be quite considerable compared to the total VVA. This means that in order to achieve a cost-efficient yet quality-focused VVA, the decision regarding the minimum viewing distance must take into consideration all the depth budget, the positions and orientations of the incoming light rays and a reasonably-selected estimated maximum observer height (i.e., the system does not necessarily need to be optimized for the tallest individual on the planet).

\footnotetext{
*https://standards.ieee.org/project/3333_1_4.html
} 


\section{RECOMMENDATIONS PER USE CASE}

In this section, we provide recommendations regarding the maximum viewing distance for each investigated use case. Based on Equation 1, we can state that

$$
R M D_{V}=\frac{D_{E}}{\tan (A R)} \times p
$$

where $R M D_{V}$ is the recommended maximum viewing distance and $p$ is a use-case-dependent variable. If it is less than 1 , then the use case requires a maximum viewing distance that is closer to the screen of the display than the default suggestion of the rule of thumb, and vice versa. For each use case, we determine an interval of $p$, which is summarized in a table at the end of the section.

Before elaborating the first use case, it needs to be noted that in certain scenarios, the general observer behavior cannot be affected; the viewing distance is more-or-less fixed, or at least it is very limited in variation. In such cases, these recommendations may provide support in choosing the appropriate angular resolution for the use case, as Equation 2 may be used to express $A R$ as

$$
A R=\arctan \left(\frac{D_{E} \times p}{R M D_{V}}\right)
$$

where $D_{E}$ is a given value, $p$ is defined by the associated recommendation and $R M D_{V}$ is determined by the constrains of the use case. In this equation, arctan is the notation of the $\tan ^{-1}$ arcus tangens inverse trigonometric function, as defined by ISO 80000-2 standard ${ }^{\dagger}$.

Finally, it needs to be separately highlighted that the recommendations in the paper are based on $A R$ and the characteristics of the use cases, while screen size and 2D-equivalent spatial resolution are not directly considered. In the aspect of manufacturing, it is easier to create a small-scale light field display with a relatively high $A R$ than to create a large one with the same $A R$. This statement already emphasizes the conflict that smaller displays are typically viewed from shorter distances, while at the same time, 3D sensation itself originates from angular ray density. In the scope of this paper, although screen size is considered in a way - among the other criteria typical to the given use case - but the recommended $p$ interval solely deals with $A R$.

\subsection{Medical imaging}

Medical use cases - such as radiology ${ }^{24}$ - are the most quality-critical, quality-dependent applications of light field technology. Any degradation of visualization quality may potentially lead to a significant loss in diagnostics accuracy. Furthermore, 3D medical imaging is often an essential component of time-sensitive medical tasks, thus no time should be wasted in such scenario on additional movements to compensate the deficiencies of 3D sensation.

If a light field display in a medical context has an $A R$ of 2 degrees - which we consider a relatively low yet adequate angular resolution in practice - then $D_{V}$ is approximately $186 \mathrm{~cm}$, according to Equation 1 . In this case, a $p$ value 0.5 in Equation 2 means that the maximum recommended viewing distance should be nearly 1 meter. If $A R$ is 0.5 - which is a high resolution - then Equation 2 with the same $p$ value results in around $372 \mathrm{~cm}$. Of course, this latter setup is not really practical in case of a small display (e.g., the size of a smaller PC monitor). Again, the $p$-interval-adjusted $R M D_{V}$ value range provides use-case-compatible maximum viewing distances, and since these are upper bounds, the actual viewing distance may be shorter. The $p$ interval we define for medical use cases runs from 0.5 to 0.8 .

\footnotetext{
${ }^{\dagger}$ https://www.iso.org/standard/64973.html
} 


\subsection{Telepresence}

Telepresence systems provide real-time audiovisual connection between individuals. While the core functionality of light field telepresence solutions is, in fact, analogous to conventional $2 \mathrm{D}$ video conferencing, they enable significantly higher levels of sense of presence, due to the natural 3D visualization of light field displays.

Light field telepresence systems may be implemented in various ways. The HoloVizio 1080T of Holografika published by Cserkaszky et al. ${ }^{25}$ - presents a large-scale, full-portrait solution, while the LightBee of Zhang et $a l .{ }^{26}$ is a self-levitating, drone-based implementation that only visualizes the head of the individual. Due to the evident diversity of telepresence systems, recommendations on the appropriate viewing distances highly depend on the types of solutions. Figure 1 shows the two prototypes during operation. Furthermore, multipurpose displays may be used as well for telepresence, as shown on Figure 2.
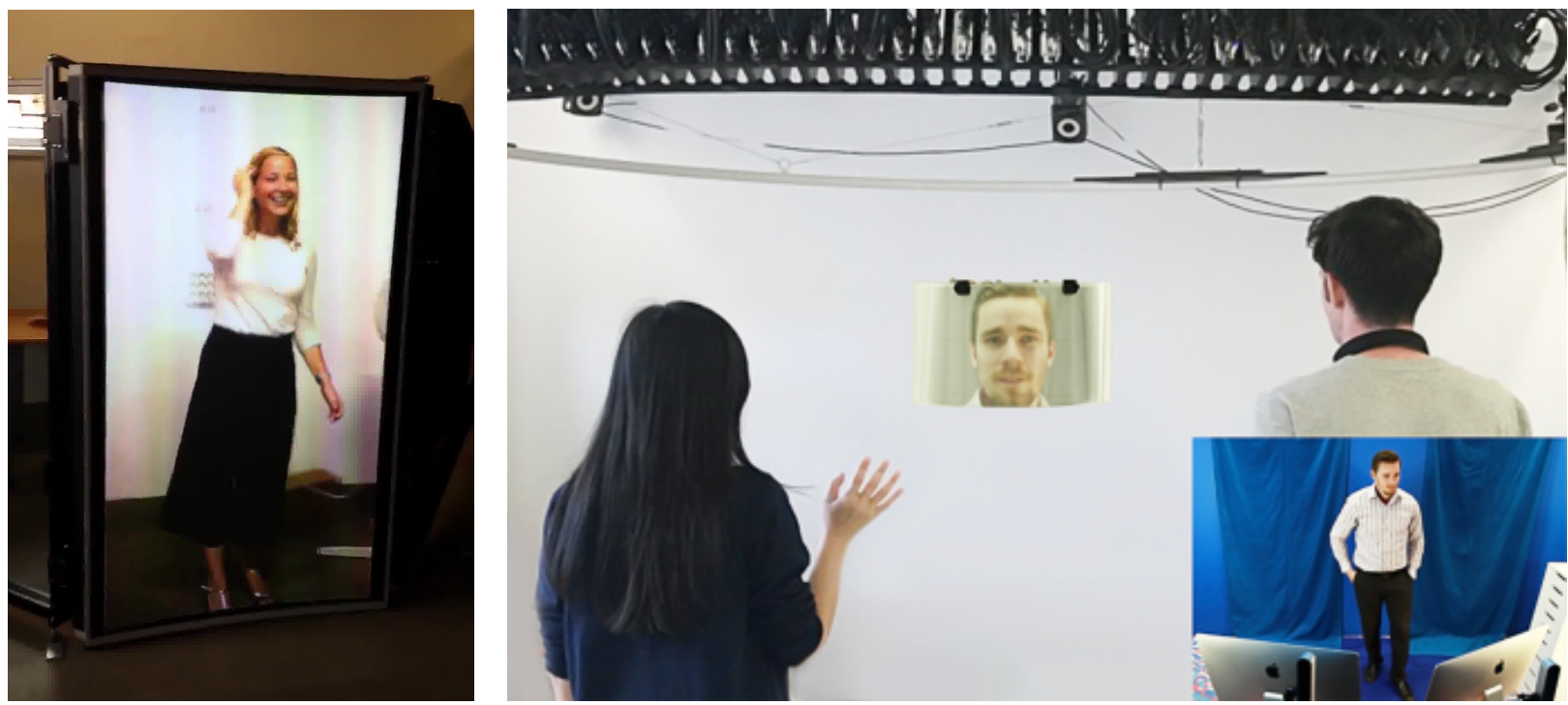

Figure 1. The HoloVizio $1080 \mathrm{~T}^{25}$ (left) and the LightBee ${ }^{26}$ telepresence systems (right).

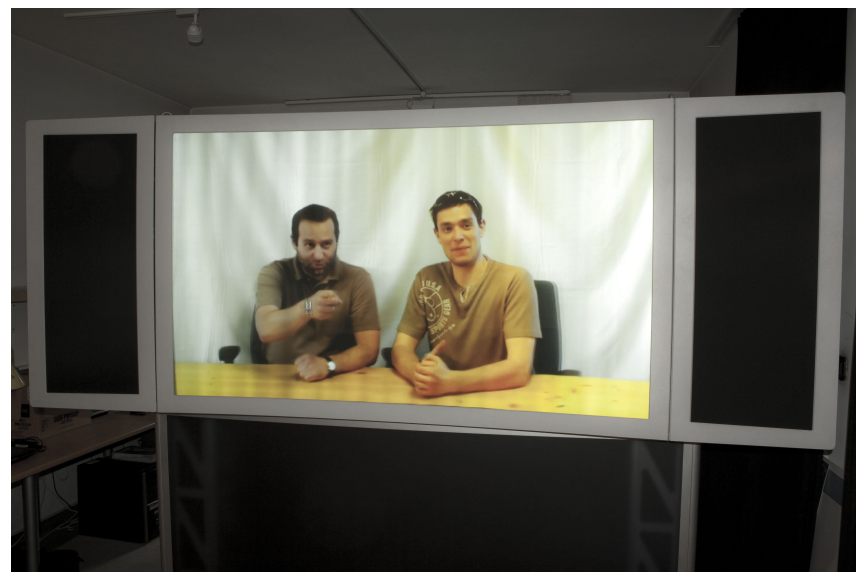

Figure 2. Experimental telepresence on a multipurpose light field display (HoloVizio HV640).

The display of the HoloVizio 1080T telepresence system - commercially installed at South Korean Telekom headquarter T-um in Seoul - has a 100-cm wide and 180-cm tall hologram screen, making it suitable to visualize the entire bodies of individuals in a true-to-scale manner. The angular resolution of the display is 0.9 degrees, and the screen provides a full-angle 180-degree FOV. Although the work does not specify the recommended viewing distance, based on Equation 1, 3D sensation is supported until $414 \mathrm{~cm}$ when considering a still observer. 
The implementation of LightBee consists of 45 projectors, has an angular resolution of 1.3 degrees and the size of the FOV is 59 degrees. The work defines the optimum viewing distance to be between $180 \mathrm{~cm}$ and $360 \mathrm{~cm}$. Unfortunately, it is not specified how this range was determined. According to Equation 1, the angular resolution of the systems results in a threshold distance at $286 \mathrm{~cm}$. During the initial subjective testing of user experience, 2 test participants simultaneously engaged in a conversation with an individual who was represented on the device, and the test participants were briefed about the confines of the active space. Although observer movement was not deterministic (i.e., any movement within the optimum viewing area was viable during the tests), it was encouraged, and with 2 test participants simultaneously occupying the area, the space for sideways movement was more than sufficient. Furthermore, the levitating device moved towards the observer the represented individual addressed during the conversation, reducing the viewing distance and introduced additional mobility in the scene.

In case of both types of light field telepresence, observation can be both static and dynamic. However, in case of drone-based solutions, the display itself may move as well, even when the observers are still. Therefore, it may be easier for such systems to support 3D sensation. It applies to both display types that telepresence systems may endure certain levels of visual degradation - including uncertainty regarding 3D sensation - since completely undistorted visualization quality is quite far from being an absolute necessity for the use case. This consideration is analogous to conventional $2 \mathrm{D}$ video conferencing applications.

Therefore, we separately provide $p$ intervals for full-body visualization and small-scale mobile systems. For large-scale systems, we recommend the value of $p$ to be between 1 and 1.5, while mobile solutions may easily endure a range from 1.2 to 2 , due to the higher level of potential respective mobility.

\subsection{Resource exploration}

The visualization of resource exploration supports the related decision making processes and it may enhance extraction planning. Unlike medical use cases, time is typically not of the essence in such context. However, visualization quality is still vital, particularly when it comes to high-value financial decisions and their practical executions (e.g., drilling).

In this use case, the number of simultaneous viewers is relatively high (e.g., everyone or at least multiple individuals who are involved in the decision-making process). The visualized content is typically carefully observed from several viewing angles across the entire FOV.

For this use case, we recommend a $p$ interval running from 0.9 to 1.4. It needs to be added that such resource exploration data at the time of this paper is relatively poor in quality and its visualization on a light field display may not necessarily strike confidence. However, as data acquisition evolves, so shall the influence of this use case in related decision-making processes become greater.

\subsection{Prototype review}

Prototype review is quite similar to the visualization of resource exploration in many aspects. It is not a timesensitive use case, yet quality is important, and the number of simultaneous viewers is high. In fact, it may even be higher, as the visualized prototype may be assessed by a wider audience (e.g., during a stakeholder meeting).

Regarding visualization requirements, prototypes may include minuscule components that should be perceived correctly in 3D. Therefore, the $A R$-based recommended maximum viewing distance is to be stricter as well, in comparison to resource exploration. Thus, we recommend that $p$ runs from 0.8 to 1.2 .

\subsection{Training and education}

Light field visualization is a potential candidate for becoming a leading tool in training and education. Training encompasses all professional types of skill enhancement and preparation (including military training), and education covers every level of public and private tuition. Regarding education, it is expected that light field technology shall emerge in a top-to-bottom manner. This means that first universities shall acquire such displays - which is already happening, but mostly with the purpose of research - followed by high schools and then elementary schools, as the market expands and the prices gradually decrease. 
The activities of training and education can be separated into two major groups. One is general education (e.g., demonstration in a high school biology class), and the other one is specialized training (e.g., unit design and validation in engineering). The core difference between them is that the latter depends slightly more on visualization quality; the loss in 3D sensation may have a greater impact. Similarly to prototype review, the visualized content during a specialized training may contain small yet detailed components that need to be perceived correctly. Therefore, while we recommend the $p$ interval to be between 0.8 and 1.5 for general education, it should be between 0.6 and 1 for specialized training.

\subsection{Gaming}

Gaming is one of the biggest markets of the century. It is simultaneously a form of recreation, entertainment, sport (with grandiose tournaments of championships), a popular content of streaming services and many more. Emerging display technologies have a vital role in making games more immersive, more engaging and thus, more enjoyable.

In the recent years, we have witnessed the rise of virtual reality (VR) on the market, and other emerging technologies - such as augmented reality (AR) - are targeting gaming as well. As an example, as soon as VR devices became affordable and mainstream, the associated gaming content became highly abundant. The same trend is expected to apply to the next generations of gaming utilities as well, including light field.

In this analysis, we only consider dedicated gaming hardware, since television-like, multipurpose, low-cost yet high-quality light field displays are not expected to enter our everyday lives in the near future. Dedicated hardware, for example, are the devices available at gaming arcades. Although in many countries, gaming arcades are disappearing due to affordable computers, consoles, smart phones and other gaming devices, the emergence of light field gaming may even revitalize arcades - of course, until the point when light field displays became affordable devices in our living rooms.

A specific type of dedicated hardware, for example, could be a table-like apparatus, for isometric gaming. Such games include classic dungeon crawlers (i.e., hack-and-slash games), real-time and turn-based strategy games and many more. Naturally, the full potential of such device can only be achieved in case of FP visualization, which would enable the player(s) to walk around the table. In case of HOP displays, a slightly-tilted screen can already serve the purpose well. An example for isometric real-time strategy gaming is shown on Figure 3.

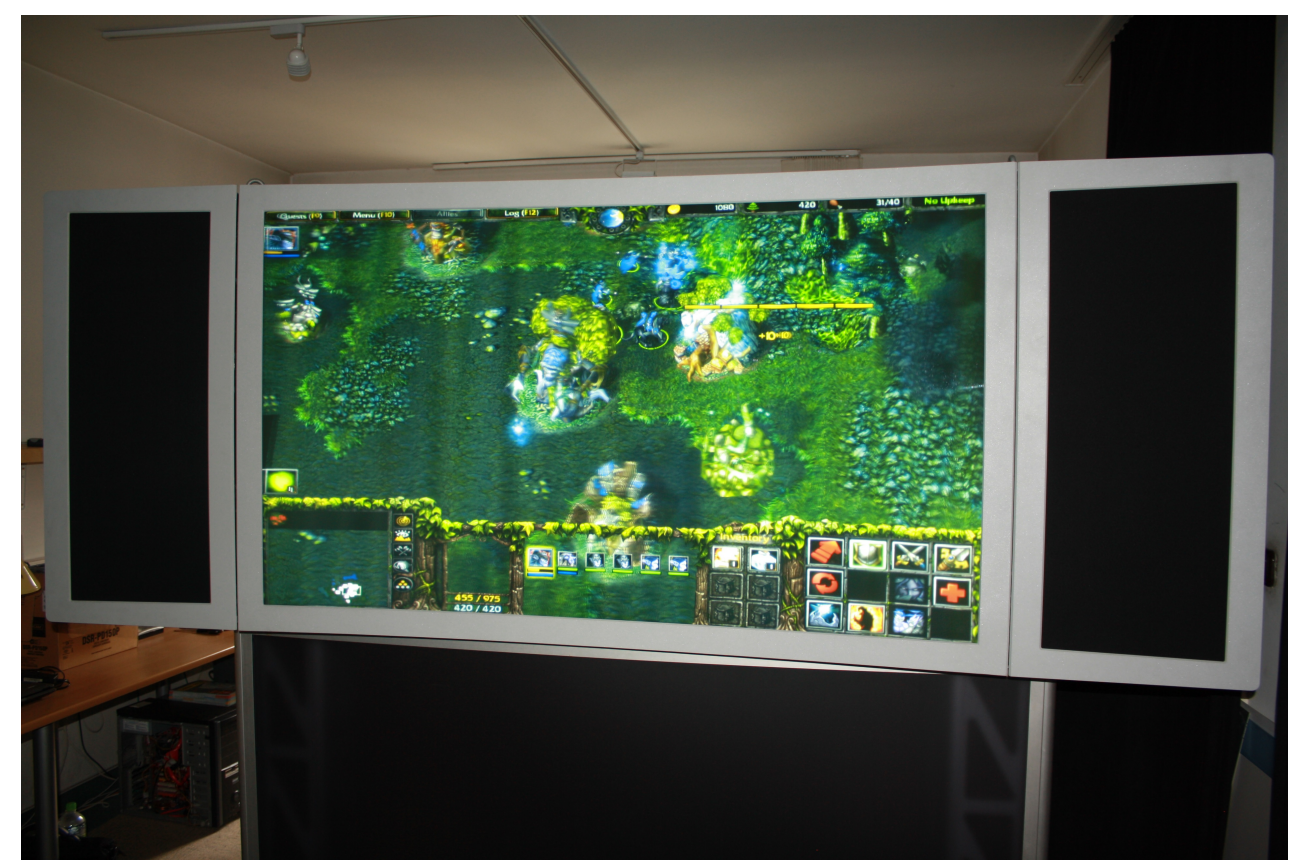

Figure 3. Warcraft III (C)Blizzard Entertainment) gameplay on the HoloVizio HV640. ${ }^{27}$ 
It is very important to note here that the novel visualization technology may inspire new gaming genres that can exploit the opportunities offered by the displays in a more efficient manner. Furthermore, the angularly selective nature of light field visualization enables independent game views for players located in different positions, which may revolutionize split-screen gaming. Yet in this case, allocating a portion of the angular domain to a specific player only reduces the potential mobility, and it does not affect the visual experience - unlike in the corresponding $2 \mathrm{D}$ gaming scenario, where 2 simultaneous players means that the game view of an individual is half of the size of the screen, which is split either vertically or horizontally.

Regarding user mobility, in case we consider conventional controls, then the player is expected to have a more-or-less unvarying position during gameplay. However, if motion sensors are utilized - e.g., during a sports game or a first-person adventure game - then the player is more mobile. This should be taken into consideration when the maximum viewing distance is recommended.

Therefore, while platforms with conventional controls should have a $p$ interval between 0.8 and 1.5, gaming systems with motion tracking should allow the same interval to scale between 1.5 and 2. Again, the actual size of the screen may demand significantly shorter viewing distances during the design of the dedicated hardware, yet it needs to be noted that the gaming community is shifting towards larger screens.

\subsection{Digital signage}

Advertising is definitely one of the biggest businesses in the world. By turning on the television or the radio, looking at "free" applications and websites on our computers and smart phones, or simply just taking a walk in the city, we are constantly being bombarded with advertisements. The game is simple: the more you grab the attention, the better chance you have at selling your products and services. This can be achieved through numerous ways, including - but, of course, not limited to - provocative content, catchy music and impressive visuals.

Such forms of utilization of immersive technologies emerge rapidly. For example, the LED façade of the SMTown Coex Atrium building $¥$ in Seoul's Gangnam District became South Korea’s biggest digital billboard in 2018. The 80-meter-wide screen projects multiple contents, including an anamorphic illusion of a crashing wave with matching sound effects. This public media art is shown on Figure 4. It was created by d'strict ${ }^{\S}$, and although it evidently carries an artistic purpose, it is not difficult to imagine the usage of this platform for advertisements with similarly immersive and eye-catching contents.

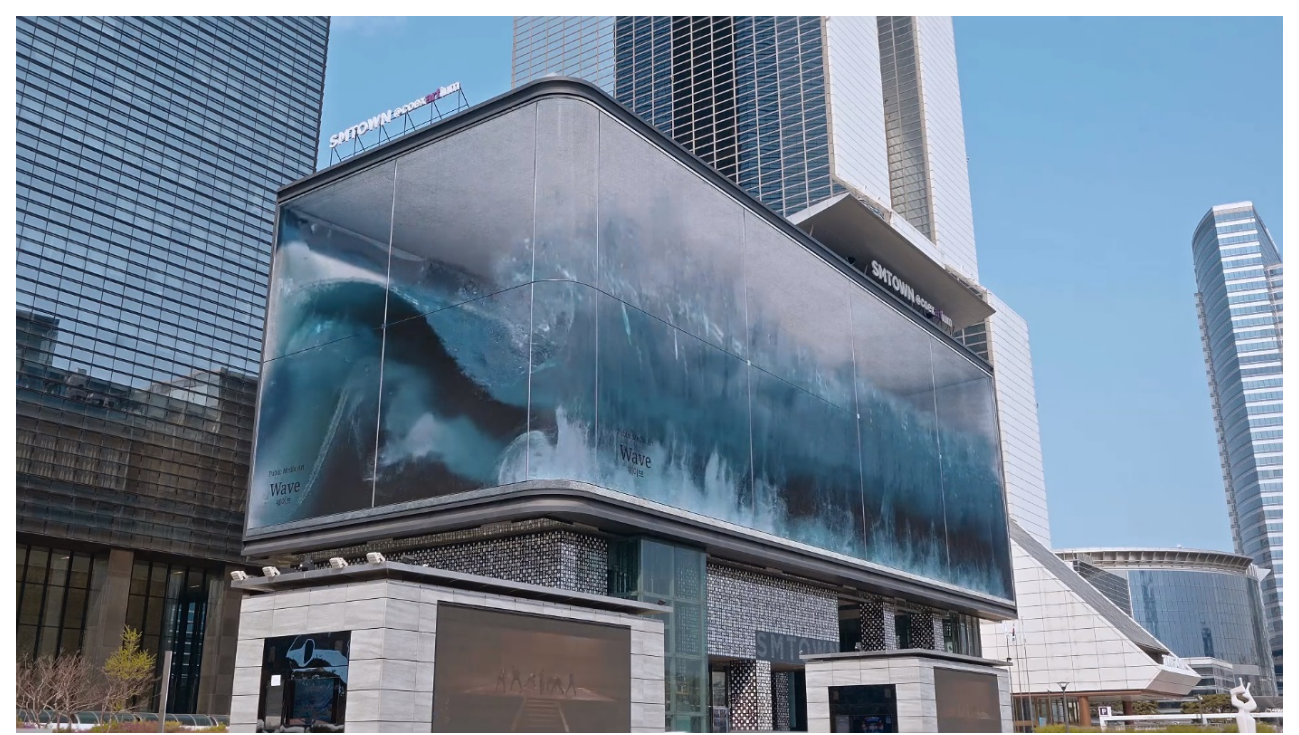

Figure 4. Public media art "Wave" at SMTown Coex Atrium. Courtesy d'strict.

\footnotetext{
${ }^{\ddagger}$ https://www.smentertainment.com/LifeStyle/CoexArtium

${ }^{\S}$ http://www.dstrict.com/arttechfactory/kr/65-Public_Media_Art_1.html
} 
Digital signage can be classified into three groups, based on size and location. The smallest ones are commonly located at the sidewalk or by a public transportation vehicle stop. They are mostly designed for pedestrians, who closely (i.e., 1 to 4 meters) walk by these portrait-oriented forms of advertisement. Billboards are larger in size, and they focus more on targeting drivers and passenger of vehicles. The dimensions may vary a lot, including bulletins and spectaculars, up to 14.63 and 18.29 meters of standard width, respectively. The third group is façade-sized signage, which is obviously not standardized, as it is the "final frontier" of signage in general, targeting anyone in its range of visibility. It is sufficient to think about the iconic digital signage of New York City's Times Square and Broadway, Piccadilly Circus in London or the 419.1-meter-long Viva Vision at Las Vegas.

Sidewalk width varies a lot globally and it also fundamentally depends on the city's local layout. The minimum width of a two-pedestrian path is around 1.5 meters, but modern layout design approaches are being shaped by user-centric research. ${ }^{28,29}$ In this highly mobile use case, a $p$ interval between 1.5 and 2.5 is recommended. At an $A R$ of 2 , a $p$ of 2.5 results in a distance above 5 meters, and nearly 19 meters at an $A R$ of 0.5 . Properly balancing $A R$ can efficiently cover wide pedestrian areas. For billboards, the $p$ interval is recommended between 3 and 5 due to the extents of vehicular mobility and the size of such signage. As for façade-sized signage, these values are elevated to 3.5 and 8 .

\subsection{Cinematography}

Compared to the other use cases addressed in this paper so far, observer behavior during scenarios of light field cinematography is quite different. Regardless of the size of the cinema, the viewer is assigned to a specific point within the area dedicated to the audience. Of course, with the rise of light field cinema, ${ }^{30}$ this model may fundamentally change, but for the scope of this analysis, let us stick to the conventional paradigm of cinema.

When purchasing a ticket to see a movie in a cinema, the price may depend on various factors, including the location of the seat. Similarly to a regular theatre, ticket prices at a movie theatre are partially determined by the position of the viewer. This is not to be confused with dynamic ticket pricing, which means that prices are influenced by the expected attendance, based on the number of sales (e.g., if a certain event is not selling well, then the ticket prices go down).

However, these prices are not purely based on the distance relative to the screen; there may be seats farther away from the screen that cost more. Yet in case of light field cinema, even if prices are not solely based on the distance, it is expected to have a significantly higher weight in the pricing model, due to the fact that 3D sensation essentially depends on it (i.e., a seat closer to the screen may provide a better visual experience).

Again, this is a use case where viewers do not move - apart from what movements are possible while sitting on the assigned seat - thus this form of compensation is rather limited, making viewers more critical to angular disturbances. ${ }^{16}$ Therefore, the recommended maximum viewing distance must be chosen accordingly. The $p$ interval should be between 0.8 and 1.2. Having a $p$ value above 1 when designing such cinema can result in the loss of 3D experience, which is generally to be avoided, yet a reasonable ticket pricing model may make it feasible.

\subsection{Cultural heritage exhibition}

Exhibitions tend to utilize state-of-the-art technologies in order to grab attention and thus make cultural heritage more appealing, more engaging. Although certain solutions particularly target younger audiences via interactive mechanisms, the visually-impressive nature of such exhibitions is meant to be enjoyed by all.

Unlike the use case of cinematography, viewers at exhibitions - e.g., in a museum or gallery - are typically highly mobile, as they slowly yet continuously walk from item to item, and depending on the outlay of the exhibited items, visitors usually observe them from multiple angles. Therefore, this is a distinctly mobile scenario, and the number of simultaneous viewers is commonly high as well - especially during popular, jam-packed exhibitions. An example of light field visualization at a museum is shown on Figure 5, which was achieved via the i-MARECULTURE project $\mathbb{I}$.

\footnotetext{
$\mathbb{I}_{\text {https: / /imareculture.eu/ }}$
} 


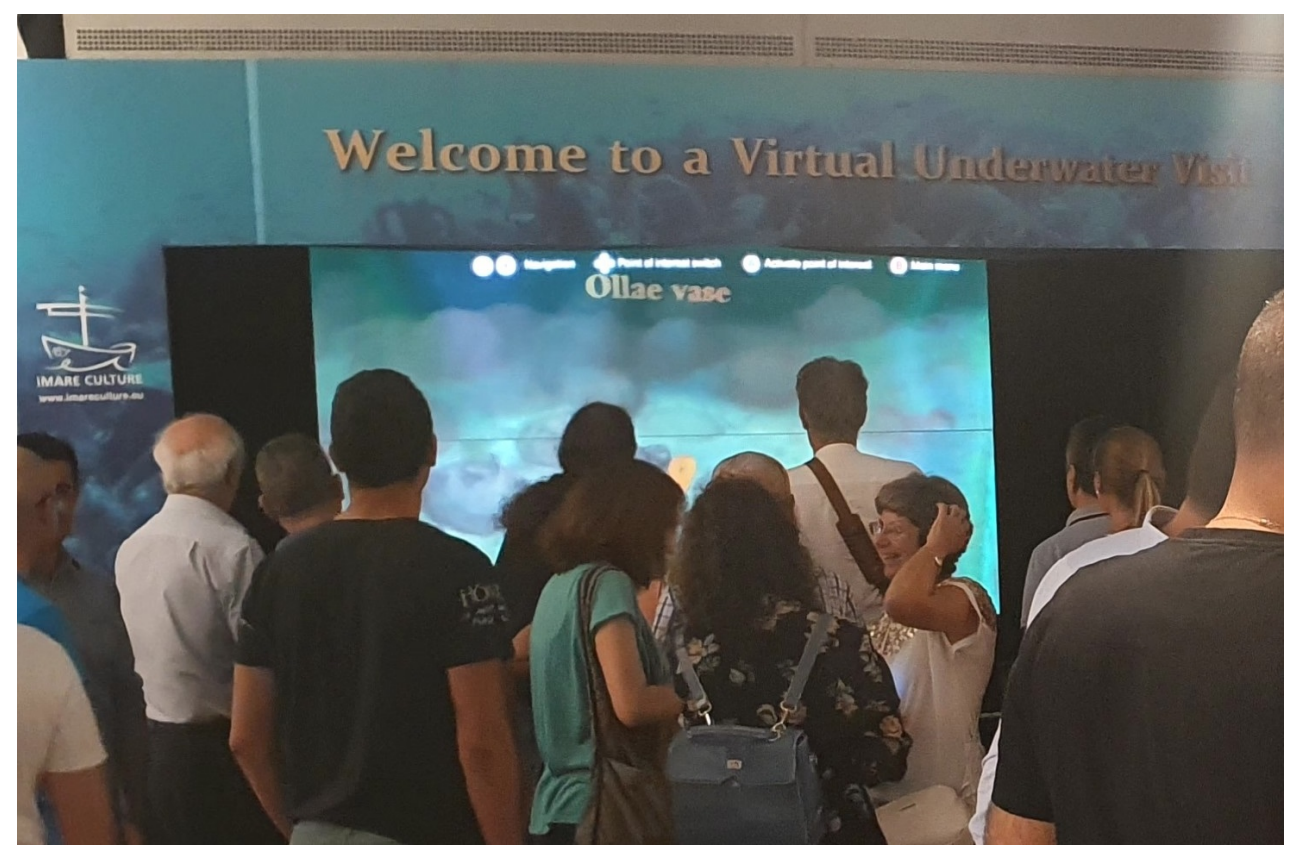

Figure 5. Artifacts of underwater cultural heritage visualized on a HoloVizio C80 light field display at the Thalassa museum (Agia Napa, Cyprus) exhibition opening on the $9^{\text {th }}$ of October, 2019.

We recommend the $p$ interval of cultural heritage exhibition to be between 0.9 and 2.5. This is a rather large interval that intends to account for the diversity of the content that is visualized in such exhibitions, and also for the diversity in observer behavior (e.g., leaning close to the content to observe its fine details versus distantly walking past the content).

\subsection{Air traffic control}

3D display technologies at air traffic controls enable a more efficient visualization of the relative distances between aircrafts and their positions in general. Since this is a safety-critical use case, quality is not to be compromised, as degraded visuals may lead to misleading, inaccurate information, the results of which can be catastrophic. The number of observers and the extents of movement are typically limited, and operators should not need to rely on additional movements to compensate the otherwise insufficient $3 \mathrm{D}$ sensation.

Due to these considerations, related recommendations should be strict and the $p$ interval should be chosen accordingly. Yet the visual details of such systems are less relevant and mostly the relative distances matter. Still, we recommend a $p$ interval between 0.7 and 0.9 due to the highly safety-critical nature of the use case.

\subsection{Driver assistance systems}

Driver assistance systems are also safety-critical applications of light field technology. One particular utilization is the light field windshield, which conveys information to the driver without the need for remove the gaze from the road. A prototype of such systems, developed by Holografika, is shown on Figure 6. This final use case of our analysis is quite unique in the sense that the physical relations (i.e., distance) between the "screen" and the eyes of the observer are very-well defined and they do not vary much over time (e.g., only via personal posture and seat settings). Therefore, the recommended $p$ interval supports more the selection of adequate $A R$ values.

As the variation in distance is low (commonly around $30 \mathrm{~cm}$, depending on the vehicle model), this should also be reflected in the size of the $p$ interval. We recommend values between 0.6 and 0.7 , ensuring a sufficiently high $A R$ for this use case (i.e., smaller $p$ values recommend better $A R$ ). As an example, if the distance between the windshield and the eyes of the driver is $100 \mathrm{~cm}$, then at a $p$ value of 0.6 , the $A R$ of the visualization system should be 2.2 degrees. 

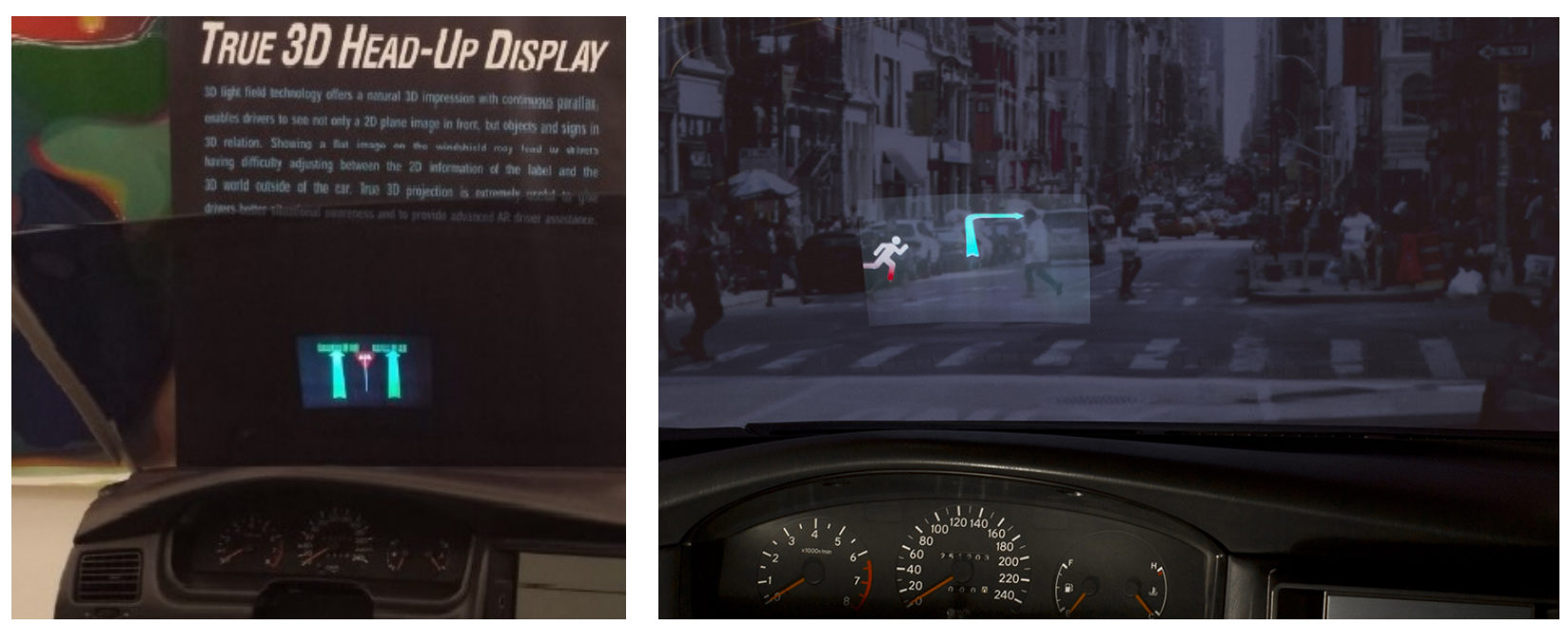

Figure 6. A prototype of a windshield-screen light field driver assistance system, developed by Holografika.

\subsection{Summary}

In Table 2, we provide a summary of the use-case-dependent $p$ intervals for the recommended maximum viewing distance.

\begin{tabular}{|l|l|}
\hline Use case & $p$ interval \\
\hline Medical imaging & $0.5-0.8$ \\
\hline Telepresence & $1-1.5$ \\
Full-body visualization & \\
Small-scale mobile systems & $1.2-2$ \\
\hline Resource exploration & $0.9-1.4$ \\
\hline Prototype review & $0.8-1.2$ \\
\hline Training and education & \\
General education & $0.8-1.5$ \\
Specialized training & $0.6-1$ \\
\hline Gaming & $0.9-1.5$ \\
Conventional controls & $1.5-2$ \\
Motion tracking & $1.5-2.5$ \\
\hline Digital signage & $3-5$ \\
Sidewalk signage & $3.5-8$ \\
Billboard & $0.8-1.2$ \\
\hline Façade-sized signage & $0.9-2.5$ \\
\hline Cinematography & $0.7-0.9$ \\
\hline Cultural heritage exhibition & $0.6-0.7$ \\
\hline Air traffic control & \\
\hline Driver assistance systems &
\end{tabular}

Table 2. Recommended $p$ interval per use case.

\section{CONCLUSION}

In this paper, we presented a set of recommendations on the viewing distance of the different utilization cases of light field visualization. The recommendations are provided in the form of intervals, which modify the value determined by the angular-resolution-based rule of thumb. We conclude that the most important factors of user-centric viewing distance selection are the potential extents of observer mobility, the detail of content and quality-critical nature of the professional context. 
The intervals introduced in this paper can be used to support the selection of adequate angular resolution values during the design of use-case-dedicated systems and to efficiently adjust the viewing environment of multipurpose displays. This can also be vital to the entry of such displays to the consumer market, as the cost-effective consideration of 3D sensation (i.e., the angular resolution for the given use case is not unnecessarily high) may play an essential role in decreasing the prices of light field displays. The next major step of device evolution shall be super resolution, which is expected to necessitate ample amounts of different types of studies, ultimately leading to recommendations.

\section{ACKNOWLEDGMENTS}

The work presented in this paper was financed by 2018-2.1.3-EUREKA-2018-00007 from the NRDI fund, Hungary. It was also funded by the European Union's Horizon 2020 research and innovation programme under the Marie Skłodowska-Curie grant agreement No 813170, and by the National Research Development and Innovation Fund based on the charter of bolster issued by the National Research Development and Innovation Office under the auspices of the Ministry for Innovation and Technology, Hungary.

\section{REFERENCES}

[1] Kara, P. A., Tamboli, R. R., Doronin, O., Cserkaszky, A., Barsi, A., Nagy, Z., Martini, M. G., and Simon, A., "The key performance indicators of projection-based light field visualization," Journal of Information Display 20(2), 81-93 (2019).

[2] Kara, P. A., Kovács, P. T., Martini, M. G., Barsi, A., Lackner, K., and Balogh, T., "Viva la Resolution: The Perceivable Differences between Image Resolutions for Light Field Displays," in [5th ISCA/DEGA Workshop on Perceptual Quality of Systems (PQS)], (2016).

[3] Kara, P. A., Tamboli, R. R., Cserkaszky, A., Martini, M. G., Barsi, A., and Bokor, L., "The Viewing Conditions of Light-Field Video for Subjective Quality Assessment," in [International Conference on 3D Immersion (IC3D)], IEEE (2018).

[4] HoloVizio 722RC large-scale 3D display. http://holografika.com/722rc/.

[5] HoloVizio C80 glasses-free 3D cinema system. http://holografika.com/c80-glasses-free-3d-cinema/.

[6] Kara, P. A., Tamboli, R. R., Cserkaszky, A., Barsi, A., Simon, A., Kusz, A., Bokor, L., and Martini, M. G., "Objective and subjective assessment of binocular disparity for projection-based light field displays," in [International Conference on 3D Immersion (IC3D)], IEEE (2019).

[7] Ahar, A., Chlipala, M., Birnbaum, T., Zaperty, W., Symeonidou, A., Kozacki, T., Kujawinska, M., and Schelkens, P., "Suitability analysis of holographic vs light field and 2D displays for subjective quality assessment of Fourier holograms," Optics Express 28(24), 37069-37091 (2020).

[8] Cserkaszky, A., Kara, P. A., Barsi, A., and Martini, M. G., "Expert Evaluation of a Novel Light-field Visualization Format," in [3DTV Conference], (2018).

[9] Cserkaszky, A., Kara, P. A., Tamboli, R. R., Barsi, A., Martini, M. G., Bokor, L., and Balogh, T., "Angularly continuous light-field format: Concept, implementation, and evaluation," Journal of the Society for Information Display 27(7), 442-461 (2019).

[10] Cserkaszky, A., Kara, P. A., Barsi, A., and Martini, M. G., "To Interpolate or not to Interpolate: Subjective Assessment of Interpolation Performance on a Light Field Display," in [International Conference on Multimedia and Expo (ICME) 8th Workshop on Hot Topics in 3D Multimedia (Hot3D)], IEEE (2017).

[11] Darukumalli, S., Kara, P. A., Barsi, A., Martini, M. G., Balogh, T., and Chehaibi, A., "Performance comparison of subjective assessment methodologies for light field displays," in [International Symposium on Signal Processing and Information Technology (ISSPIT)], 28-33, IEEE (2016).

[12] Dricot, A., Jung, J., Cagnazzo, M., Pesquet, B., Dufaux, F., Kovács, P. T., and Adhikarla, V. K., "Subjective evaluation of super multi-view compressed contents on high-end light-field 3D displays," Signal Processing: Image Communication 39, 369-385 (2015).

[13] Jones, A., McDowall, I., Yamada, H., Bolas, M., and Debevec, P., "Rendering for an interactive $360^{\circ}$ light field display," ACM Transactions on Graphics 26(3) (2007). 
[14] Kara, P. A., Cserkaszky, A., Martini, M. G., Barsi, A., Bokor, L., and Balogh, T., "Evaluation of the Concept of Dynamic Adaptive Streaming of Light Field Video," IEEE Transactions on Broadcasting 64(2), 407-421 (2018).

[15] Kara, P. A., Tamboli, R. R., Cserkaszky, A., Martini, M. G., Barsi, A., and Bokor, L., "The perceived quality of light-field video services," in [Applications of Digital Image Processing XLI], SPIE (2018).

[16] Kara, P. A., Cserkaszky, A., Darukumalli, S., Barsi, A., and Martini, M. G., "On the Edge of the Seat: Reduced Angular Resolution of a Light Field Cinema with Fixed Observer Positions," in [9th International Conference on Quality of Multimedia Experience (QoMEX)], IEEE (2017).

[17] Kara, P. A., Kovács, P. T., Martini, M. G., Barsi, A., Lackner, K., and Balogh, T., "From a Different Point of View: How the Field of View of Light Field Displays affects the Willingness to Pay and to Use," in [8th International Workshop on Quality of Multimedia Experience (QoMEX)], IEEE (2016).

[18] Kawakita, M., Iwasawa, S., Sakai, M., Haino, Y., Sato, M., and Inoue, N., "3D image quality of 200-inch glasses-free 3D display system," in [Stereoscopic Displays and Applications XXIII], SPIE (2012).

[19] Lee, C.-K., Park, S.-g., Moon, S., Hong, J.-Y., and Lee, B., "Compact multi-projection 3D display system with light-guide projection," Optics express 23(22), 28945-28959 (2015).

[20] Petrov, N. I., Khromov, M. N., and Sokolov, Y. M., "Large-screen multi-view 3D display," OSA Continuum 2(9), 2601-2613 (2019).

[21] Tamboli, R. R., Vupparaboina, K. K., Ready, J., Jana, S., and Channappayya, S., "A subjective evaluation of true 3D images," in [International Conference on 3D Imaging (IC3D)], IEEE (2014).

[22] Tamboli, R. R., Appina, B., Channappayya, S., and Jana, S., "Super-multiview content with high angular resolution: 3D quality assessment on horizontal-parallax lightfield display," Signal Processing: Image Communication 47, 42-55 (2016).

[23] Tamboli, R. R., Appina, B., Channappayya, S., and Jana, S., "Achieving high angular resolution via view synthesis: quality assessment of 3D content on super multiview lightfield display," in [International Conference on 3D Immersion (IC3D)], IEEE (2017).

[24] Cserkaszky, A., Kara, P. A., Barsi, A., and Martini, M. G., "The potential synergies of visual scene reconstruction and medical image reconstruction," in [Novel Optical Systems Design and Optimization XXI], SPIE (2018).

[25] Cserkaszky, A., Barsi, A., Nagy, Z., Puhr, G., Balogh, T., and Kara, P. A., "Real-time light-field 3D telepresence," in [7th European Workshop on Visual Information Processing (EUVIP)], IEEE (2018).

[26] Zhang, X., Braley, S., Rubens, C., Merritt, T., and Vertegaal, R., "Lightbee: A self-levitating light field display for hologrammatic telepresence," in [CHI Conference on Human Factors in Computing Systems], (2019).

[27] Cserkaszky, A., Kara, P. A., Tamboli, R. R., Barsi, A., Martini, M. G., and Balogh, T., "Light-field capture and display systems: limitations, challenges, and potentials," in [Novel Optical Systems Design and Optimization XXI], SPIE (2018).

[28] Kim, S., Choi, J., and Kim, Y., "Determining the sidewalk pavement width by using pedestrian discomfort levels and movement characteristics," KSCE Journal of Civil Engineering 15(5), 883-889 (2011).

[29] Lee, B. J., Jang, T. Y., Wang, W., and Namgung, M., "Design criteria for an urban sidewalk landscape considering emotional perception," Journal of urban planning and development 135(4), 133-140 (2009).

[30] Kara, P. A., Martini, M. G., Nagy, Z., and Barsi, A., "Cinema as large as life: Large-scale light field cinema system," in [International Conference on 3D Immersion (IC3D)], IEEE (2017). 